\title{
SIMDiscovery: a simulation-based preparation program for adolescents undergoing spinal fusion surgery
}

\author{
Lauren M. Potthoff ${ }^{1,7,8}$ (C) Michael P. Glotzbecker ${ }^{2,7} \cdot$ Brianna O'Connell $^{3,4} \cdot$ Nora P. O'Neill $^{2} \cdot$ Kelsey M. Graber ${ }^{3} \cdot$ \\ Carrie A. Byrne ${ }^{4,5}$. Joseph M. Tremmel ${ }^{2}$. Peter Weinstock ${ }^{3,6,7} \cdot$ Lauren Mednick $^{1,3,7}$
}

Received: 9 April 2020 / Accepted: 22 February 2021 / Published online: 10 March 2021

(c) Scoliosis Research Society 2021

\begin{abstract}
Purpose Spinal fusion surgery is associated with high levels of stress and anxiety for patients and their caregivers. Medical simulation has demonstrated efficacy in improving preparedness, knowledge, and overall experience prior to other medical procedures. The current study examines the utility of a multi-faceted preparation program (SIMDiscovery) using simulation techniques to reduce anxiety and increase preparedness among patients undergoing spinal fusion surgery and their caregivers. Methods Participants attended SIMDiscovery where they received hands-on preparation about what to expect before, during, and after their surgery. Anxiety, preparedness, and knowledge about the procedure were assessed pre- and post-participation using self-report measures. Participants also completed a questionnaire at their first post-operative medical appointment. Differences from pre to post and between patients and caregivers were calculated with paired and independent sample $t$-tests. Results Participants included 22 patients and 29 caregivers. Post-SIMDiscovery, both groups demonstrated increased knowledge for the surgical process and lower state anxiety. Patients reported increased feelings of preparedness in all areas while caregivers reported increased feelings of preparedness in most areas. Families continued to report positive impact of the program 30 days after surgery; however, they also identified areas where they desired increased preparation.

Conclusions SIMDiscovery increased patients' and caregivers' knowledge regarding spinal fusion surgery and helped them feel less anxious and more prepared regarding most aspects of the surgical process. These changes were generally maintained throughout the post-operative period. Participants identified areas for increased preparation, highlighting the importance of continuing to adapt programs based on patient and family feedback.
\end{abstract}

Level of evidence Level III.

Keywords Pediatric $\cdot$ Scoliosis $\cdot$ Posterior spinal fusion $\cdot$ Simulation-based $\cdot$ Preparation

\section{Introduction}

Posterior spinal fusion (PSF) is the most common surgical intervention to treat adolescent idiopathic scoliosis (AIS)

Lauren M. Potthoff and Michael P. Glotzbecker are co-first authors.

[1]. PSF is associated with high levels of stress and anxiety

Lauren M. Potthoff

lmpotthoff@luriechildrens.org

1 Department of Psychiatry, Boston Children's Hospital, Boston, MA, US

2 Department of Orthopedic Surgery, Boston Children's Hospital, Boston, MA, US

3 Boston Children's Hospital Simulator Program, Boston, MA, US

4 Department of Child Life, Boston Children's Hospital, Boston, MA, US
5 Boston Children's Hospital Perioperative Program, Boston, MA, US

6 Department of Anesthesia, Critical Care and Pain Medicine, Boston Children's Hospital, Boston, MA, US

7 Harvard Medical School, Boston, MA, US

8 Ann and Robert H. Lurie Children's Hospital of Chicago, Chicago, IL, USA 
in both patients [2, 3] and their caregivers [4], which may negatively impact postoperative recovery [5]. Participation in pre-operative preparation programs has been shown to reduce feelings of stress and anxiety in pediatric patients and their caregivers and to improve medical outcomes for many different medical and surgical interventions [6, 7]. Only one study has examined the use of preoperative preparation programs in reducing anxiety among patients undergoing PSF. Using a randomized control study design, Rhodes et al., (2015) compared self-reported anxiety among families who participated in standard care preparation with families who received standard care preparation, plus a 30-min hospital tour [8]. Findings indicate that self-reported anxiety did not significantly differ between intervention and control groups for patients at three of the four time points. No significant differences in anxiety were found between caregiver groups.

Importantly, it is possible that the researchers did not find significant differences between groups because the intervention (i.e., hospital tour) lacked an enhanced experiential component. Such preparation methods may be insufficient to achieve full and successful knowledge and skill transfer [9] as they do not include opportunities to learn through active practice, realistic exposure, and rehearsal. Further, this study did not include a focus on preparing families for what to expect post-operatively, specifically when returning home. Having realistic expectations for what patients may experience before, during, and after a procedure-including at-home recovery-is an essential part of preparation [10].

The current study aimed to examine the impact of a comprehensive experiential preparation program for patients and caregivers scheduled for PSF (SIMDiscovery). The program relied on simulation teaching methodologies and comprehensive preparation at all stages of the surgical experience. We hypothesized that (1) caregivers' and patients' performances on a PSF knowledge questionnaire would improve after SIMDiscovery; (2) patients and caregivers would report less anxiety and increased preparedness after SIMDiscovery; and (3) improved feelings of preparedness would be maintained throughout the post-operative period.

\section{Materials and methods}

This prospective study was conducted at a single tertiary care pediatric hospital by a research team consisting of members of the Psychiatry, Simulator, Orthopedic Surgery, and Child Life Services departments. The study was registered by the US National Library of Medicine's Clinical Trials website, as it was categorized as a behavioral intervention study. Recruitment occurred between February and June 2019 after obtaining institutional IRB approval. Follow-up data was collected until the last patient was 30 days post-surgery.
SIMDiscovery-orthopedics is a group simulation program developed as part of a hospital-based simulation service line for patients and caregivers. SIMDiscovery is a multi-faceted preparation program that utilizes simulation techniques and hands-on, experiential opportunities (e.g., interacting with medical equipment, practicing technique for getting out of bed following surgery) for patients who will undergo PSF and their families. During SIMDiscovery, patients and caregivers are guided through a simulated hospital setting which includes nine stations designed to mirror the stages of the perioperative process (see Table 1). Stations provide families with education and experiential learning opportunities regarding what they can anticipate before, during, and after the surgery. Station content was created by medical and psychosocial experts in each area.

\section{Eligibility, screening, and recruitment}

Patients scheduled for PSF between February and August 2019 were screened for eligibility. Inclusion criteria included: at least 10 years of age on the day of SIMDiscovery, diagnosis of AIS, and ability to respond to questionnaires in English. Exclusion criteria included: other significant chronic medical conditions, anterior surgical access, intensive care unit stay expected, and significant cognitive delays or intellectual disabilities.

To maximize participation in this new program, we offered SIMDiscovery to all eligible patients within a geographic area. Families received a letter in the mail describing SIMDiscovery and were then contacted by a member of the Simulator Program via phone or email to assess interest in attending. Families interested in SIMDiscovery subsequently received a postcard in the mail introducing the research study. Research assistants later contacted families via phone to offer study participation. When a family could not be reached prior to attending SIMDiscovery, research staff approached them in person when they arrived.

Eighty families were contacted to participate in SIMDiscovery and 34 families attended. Families who declined SIMDiscovery identified schedule conflicts, barriers associated with traveling to the hospital, or applicability of the program. Twenty-eight of the 34 patients were eligible for the study, and 22 agreed to participate. See Fig. 1 detailing participant recruitment. Informed consent for caregivers and patients $\geq 18$ years old and assent for patients $<18$ years old was obtained the day of SIMDiscovery prior to completing study measures.

\section{Study measures}

Prior to and immediately after participating in SIMDiscovery, patients and at least one caregiver completed questionnaires electronically via REDCap [11]. If a second caregiver 
Table 1 SIMDiscovery Stations

\begin{tabular}{|c|c|c|}
\hline Stations & Station elements & Multidisciplinary team members \\
\hline Pre-op admitting & Consent forms-any blood/urine tests & $\begin{array}{l}\text { Pre-op admitting nurse } \\
\text { Practitioner } \\
\text { Child life specialist }\end{array}$ \\
\hline Pre-op holding & $\begin{array}{l}\text { Changing into hospital gown } \\
\text { Roles of surgical team members } \\
\text { Peripheral intravenous line placement with numbing agent } \\
\text { Pre-surgical medication }\end{array}$ & $\begin{array}{l}\text { Pre-op holding nurse } \\
\text { Child life specialist }\end{array}$ \\
\hline Operating room $(\mathrm{OR})$ & $\begin{array}{l}\text { Anesthesia process } \\
\text { Role of the operating room nurse } \\
\text { Surgical equipment used (including rods) }\end{array}$ & $\begin{array}{l}\text { Operating room nurse } \\
\text { Anesthesiologist } \\
\text { Child life specialist }\end{array}$ \\
\hline Post-anesthesia care unit (PACU) & $\begin{array}{l}\text { Medical equipment } \\
\text { Parental presence } \\
\text { Pain management }\end{array}$ & $\begin{array}{l}\text { Post anesthesia care unit nurse } \\
\text { Child life specialist }\end{array}$ \\
\hline Inpatient experience & $\begin{array}{l}\text { Anticipated pain } \\
\text { Pain management } \\
\text { Available resources/services in hospital }\end{array}$ & $\begin{array}{l}\text { Inpatient nurse } \\
\text { Child life specialist }\end{array}$ \\
\hline Dressing changes & $\begin{array}{l}\text { Dressing change demonstration } \\
\text { Wound care } \\
\text { Medical materials used (e.g. gauze/bandages) }\end{array}$ & $\begin{array}{l}\text { Orthopedic nurse practitioner } \\
\text { Child life specialist }\end{array}$ \\
\hline Physical therapy and mobility & $\begin{array}{l}\text { Physical therapy expectations } \\
\text { Exercises } \\
\text { Timeline for return to activity at home }\end{array}$ & $\begin{array}{l}\text { Physical therapist } \\
\text { Child life specialist }\end{array}$ \\
\hline Meet the surgeon and hardware & $\begin{array}{l}\text { Question/answer session with surgeon } \\
\text { Spinal model with fusion hardware }\end{array}$ & $\begin{array}{l}\text { Orthopedic surgeon } \\
\text { Child life specialist }\end{array}$ \\
\hline Discharge & $\begin{array}{l}\text { Discharge expectations } \\
\text { Prescribed medications and potential side effects } \\
\text { Follow up care instructions }\end{array}$ & $\begin{array}{l}\text { Orthopedic physician assistant } \\
\text { Orthopedic nurse practitioner } \\
\text { Social worker } \\
\text { Child life specialist } \\
\text { Previous patient/parent }\end{array}$ \\
\hline
\end{tabular}

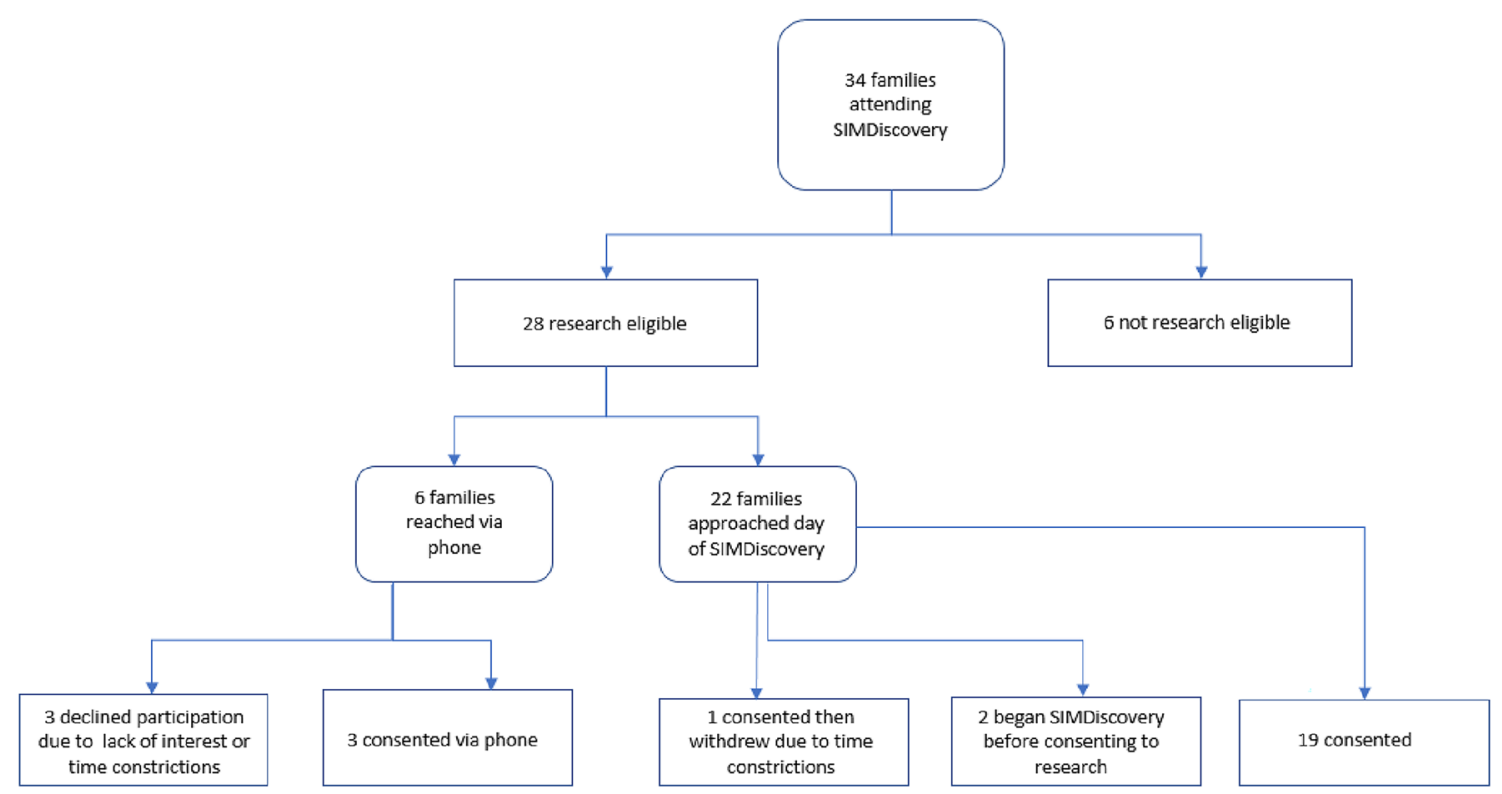

Fig. 1 Participant recruitment 
attended and wanted to participate in the study, they also completed questionnaires. Participants completed a questionnaire at their first post-operative visit approximately 30 days after surgery. See Table 2 for questionnaires completed at each timepoint.

\section{Demographics questionnaire}

Caregivers completed a brief questionnaire providing demographic information as well as medical and psychiatric history.

\section{Knowledge assessment}

All participants completed a 10-question knowledge assessment created for this study (see online Appendix A). Content was provided by medical and psychosocial staff familiar with the PSF perioperative experience and includes six true/false and four multiple choice questions. Scores on this measure ranged from 0 to 10 (e.g., answering all 10 questions correctly equals a score of 10$)$.

\section{Anxiety measures}

State/trait anxiety: caregivers and patients over age 15 completed the state/trait anxiety inventory (STAI) [12] to assess state anxiety (current emotional state) and trait anxiety (general proneness to anxious behavior). Patients under age 15 completed the State/Trait Anxiety Inventory for Children (STAIC) [13]. The State Anxiety items were completed preand post-SIMDiscovery, while the Trait Anxiety items were only completed prior to participation. These measures are well-validated [13, 14].

Visual analog scale (VAS): anxiety regarding PSF was assessed for all participants with one item using a 10-point VAS [0 (not at all anxious), 10 (very anxious)]. The VAS is highly valid and reliable with children, adolescents, and adults $[15,16]$.

\section{Preparedness measures}

\section{Preparedness}

Caregiver preparedness was measured by the caregiver preparedness scale (CPS) [17], which asks caregivers to rate on a scale of $0-4$ (not at all prepared to very prepared) how prepared they feel to care for eight areas of the patient's physical and emotional needs. We modified the CPS to assess patients' perception of their ability to care for or advocate for themselves in 6 different areas of physical and emotional needs (See online Appendix B) [17].

\section{Visual analog scale}

Visual analog scale preparedness was assessed for all participants with three items using a 10-point VAS $[15,16]$. These questions assessed feelings of preparedness [0 (not at all prepared), 10 (very well prepared)] regarding the pre-operation holding experience, inpatient experience, and returning home. Participants were also asked to complete these three items at their 30 day post-operative visit.

\section{Follow-up questionnaire}

In addition to completing the VAS preparedness items at the 30-day postoperative visit, families also provided written feedback about their overall experience with surgery and SIMDiscovery.

\section{Statistical analysis}

Paired samples $t$ tests were used to analyze patients' and caregivers' pre and post-SIMDiscovery STAI/STAI-C, VAS
Table 2 SIMDiscovery Group Study Measures Timeline

\begin{tabular}{|c|c|c|c|c|c|c|}
\hline & \multicolumn{2}{|c|}{ Pre-SIMDiscovery } & \multicolumn{2}{|c|}{ Post-SIMDiscovery } & \multicolumn{2}{|c|}{$\begin{array}{l}\text { 30-day postopera- } \\
\text { tive visit }\end{array}$} \\
\hline & Caregiver & Patient & Caregiver & Patient & Caregiver & Patient \\
\hline Demographics & $\mathrm{X}$ & & & & & \\
\hline Knowledge assessment & $\mathrm{X}$ & $\mathrm{X}$ & $\mathrm{X}$ & $\mathrm{X}$ & & \\
\hline \multicolumn{7}{|l|}{ Anxiety measures } \\
\hline Trait anxiety inventory & $\mathrm{X}$ & $\mathrm{X}$ & & & & \\
\hline State anxiety inventory & $\mathrm{X}$ & $\mathrm{X}$ & $\mathrm{X}$ & $\mathrm{X}$ & & \\
\hline VAS for anxiety & $\mathrm{X}$ & $\mathrm{X}$ & $\mathrm{X}$ & $\mathrm{X}$ & & \\
\hline \multicolumn{7}{|l|}{ Preparedness measures } \\
\hline Caregiver/child preparedness scale & $\mathrm{X}$ & $\mathrm{x}$ & $\mathrm{x}$ & $\mathrm{X}$ & & \\
\hline VAS for preparedness & $\mathrm{X}$ & $\mathrm{X}$ & $\mathrm{X}$ & $\mathrm{X}$ & $\mathrm{X}$ & $\mathrm{X}$ \\
\hline Follow-up questionnaire & & & & & $\mathrm{X}$ & $\mathrm{X}$ \\
\hline
\end{tabular}


responses, CPS, and knowledge assessment scores. Paired samples $t$ tests were also conducted to examine feelings of preparedness from immediately after SIMDiscovery to the 30-day postoperative visit. Independent sample $t$ tests were used to compare caregivers to patients on the same measures. Bivariate analyses were conducted to examine relevant medical or demographic variables to control for in analyses; none were significant. Statistical analyses were performed in SPSS Version 24.0.0.0.

\section{Results}

Twenty-two patients and their caregivers participated in the study during the three SIMDiscovery programs in 2019. Caregivers included 19 mothers, nine fathers, and one legal guardian; seven patients had two caregivers participate. The mean age of the patients was 14.6 years (range 11-19 years). The majority of patients identified as female (59\%) and Caucasian (83\%). Eight patients underwent a prior surgery although none had previously experienced an orthopedic surgery; three patients reported a prior medical hospitalization. One participant found the experience overwhelming and did not complete post-SIMDiscovery measures; they were excluded from analyses. All participants completed the post-operative follow-up questionnaire.

\section{Knowledge}

Knowledge about the spinal fusion surgery increased for patients and caregivers after participating in SIMDiscovery [patients $t(21)=-3.8, p=0.001$; caregivers $t(27)=-4.3$, $p=<0.001]$.

\section{Anxiety}

On the STAI and STAI-C, patients and caregivers evidenced lower state anxiety following participation in SIMDiscovery compared with prior to participation [patients (under 15) $t(11)=3.1, p=0.01$; patients (over 15) $t(7)=2.8, p=0.27$; caregivers $t(26)=5.0, p=<0.001]$. Before SIMDiscovery, patients ages 15 and above reported their state anxiety to be consistent with their trait anxiety $\left(M_{\text {state }}=44.1\right.$, $\left.\mathrm{SE}=4.5 ; M_{\text {trait }}=43.5, \mathrm{SE}=4.00\right)$. After SIMDiscovery, older patients reported significantly lower state anxiety $(M=34.6, \mathrm{SE}=2.7)$ than their trait anxiety, $t(7)=-2.8$, $p=0.03$. Caregivers reported their state anxiety $(M=37.7$, $\mathrm{SE}=2.1)$ to be significantly higher than their trait anxiety $(M=32.2, \mathrm{SE}=1.4)$ before SIMDiscovery $t(26)=3.4$, $p=0.002$; no significant differences between caregiver state and trait anxiety were observed after program participation.

On the VAS assessing feelings of anxiety when thinking about the patient's upcoming PSF, caregivers endorsed feeling significantly less anxious after SIMDiscovery $(M=4.2, \mathrm{SE}=0.5)$ than before $(M=6.9, \mathrm{SE}=0.4)$, $t(25)=4.2, p<0.05$. Among patients, this difference approached statistical significance $[M=4.9, \mathrm{SE}=0.7$; $M=3.9, \mathrm{SE}=0.7 ; t(19)=2.1, p=0.049]$.

\section{Preparedness}

Patients reported increased feelings of preparedness in all six areas after SIMDiscovery $(p=<0.001-0.024)$. Caregivers similarly reported increased feelings of preparedness in all areas ( $p=0.004-0.02)$ except when thinking about the stress of caring for their child following surgery $(p=0.119)$.

On the VAS, caregivers reported feeling significantly more prepared for the patient's pre-operation holding experience $[M=7.8, \mathrm{SE}=0.4 ; t(26)=-3.4, p<0.05]$, inpatient hospital stay $[M=7.9, \mathrm{SE}=0.4 ; t(26)=-3.6, p<0.05]$, and returning home $[M=7.7, \mathrm{SE}=0.4 ; t(26)=-3.9, p<0.05]$ after SIMDiscovery than before $(M=5.6, \mathrm{SE}=0.6 ; M=5.5$, $\mathrm{SE}=0.6 ; M=5.4, \mathrm{SE}=0.5$, respectively). These differences were also observed among patients $[M=7.1, \mathrm{SE}=0.5$; $t(19)=-2.2, p=0.041 ; M=7.7, \mathrm{SE}=0.5 ; t(19)=-3.3$, $p=0.003 ; M=7.8, \mathrm{SE}=0.5 ; t(19)=-3.3, p=0.004]$.

\section{Postoperative follow-up}

Patients' increased feelings of preparedness remained consistent; no significant differences in reported feelings of preparedness from immediately after SIMDiscovery to the 30-day follow-up were found for pre-operation holding $(M=6.9, \mathrm{SE}=0.7 ; M=7.6, \mathrm{SE}=0.6)$, inpatient $(M=7.6$, $\mathrm{SE}=0.8 ; M=7.5, \mathrm{SE}=0.7)$, or returning home $(M=7.9$, $\mathrm{SE}=0.6 ; M=7.3, \mathrm{SE}=0.7)$.

Caregivers, on average, reported feeling significantly more prepared for pre-operation holding $(M=9.4, \mathrm{SE}=0.2)$ compared with how prepared they felt for that experience immediately after SIMDiscovery $(M=7.5, \mathrm{SE}=0.7)$, $t(12)=-3.1, p=0.009$. Average feelings of preparedness for the inpatient stay and returning home remained consistent across timepoints (inpatient stay: $M=7.6, \mathrm{SE}=0.67$; $M=9.1, \mathrm{SE}=0.3$; returning home: $M=7.3, \mathrm{SE}=0.7$; $M=7.8, \mathrm{SE}=0.7)$.

\section{Discussion}

PSF is a common intervention for AIS and is associated with increased stress and anxiety for patients and their caregivers. Pre-surgical preparation programs are effective in reducing both stress and anxiety, yet few studies exist examining the benefits of these programs among patients undergoing orthopedic surgeries and their caregivers. As hypothesized, patient and caregiver knowledge regarding the procedure 
increased following participation in the program. Consistent with prior studies among other medical groups [18], results demonstrated decreased anxiety among patients and caregivers following SIMDiscovery. In contrast to the findings of Rhodes et al., (2015), our results may be attributed to the experiential component that SIMDiscovery offers. Rhodes et al., (2015) implemented a 30-min hospital tour as their intervention while SIMDiscovery incorporates opportunities to learn about the perioperative process while interacting with equipment and providers. Thus, our findings support the idea that hands-on opportunities for learning are beneficial components to preparation programs.

Patients indicated that they felt increased preparedness in all areas assessed after participating in SIMDiscovery while caregivers endorsed increased preparedness in nearly every area: caregivers did not report a statistically significant increase in preparedness when asked about the stress of caring for the child following surgery. This finding may highlight that despite increased preparation and knowledge regarding the procedure and its after-effects, caregivers continue to anticipate experiencing stress during the postoperative period potentially due to the complicated nature of the surgical procedure and recovery.

Feelings of preparedness were generally maintained immediately after participation in SIMDiscovery to the first post-operative appointment 30 days after surgery. Patients' improved feelings of preparedness remained consistent for all three areas. At the 30-day follow-up, caregivers reported feeling more prepared for the pre-operation holding experience and similarly prepared for returning home compared with immediately after SIMDiscovery, supporting the notion that the experiential and interactive components of the program may contribute to these maintained feelings of preparedness.

Despite these significant improvements in anxiety and preparedness, on the 30-day post-operative follow-up questionnaire, some participants identified continued concerns. They most commonly endorsed wanting increased preparation around pain management following surgery (i.e., "How to deal with pain if you cannot take any more meds") and how to continue caring for the patient/themselves at home (i.e., "More about recovery a little further out than the first week.") These qualitative data highlight further aspects in which patients and their caregivers desire increased preparation. This feedback is beneficial as SIMDiscovery is a new and evolving program. Additional information in these areas can be integrated into the program to better address these concerns. Further, medical teams can utilize these data to better prepare families by providing increased information about expectations in the weeks after discharge.

Please refer to Table 3 for information on the actual and opportunity costs associated with SIMDiscovery as well as the key stakeholders involved in each aspect of program development and implementation. This description provides a framework of the staff required to plan and implement SIMDiscovery at this particular institution; however, it would be feasible to adjust the number of participants based on staffing resources available in other institutions.

Table 3 Personnel and Time Required for Program Creation and Implementation

\begin{tabular}{|c|c|c|c|}
\hline Task & Time spent & Multidisciplinary team members & Goal/outcome \\
\hline \multicolumn{4}{|l|}{ Program creation } \\
\hline Program planning meeting & $1 \mathrm{~h}$ & $\begin{array}{l}\text { Physician } \\
\text { Child life specialist } \\
\text { Physician's assistant } \\
\text { Pre-operation clinic nurse } \\
\text { Pre-operation holding nurse } \\
\text { Inpatient floor nurse }\end{array}$ & $\begin{array}{l}\text { Identified and finalized: stations to include in SIMDis- } \\
\text { covery } \\
\text { Additional staff representatives for the stations }\end{array}$ \\
\hline Staff participation recruitment & $8 \mathrm{~h}$ & Simulation center staff & $\begin{array}{l}\text { Coordinated with representatives from different depart- } \\
\text { ments regarding participation in SIMDiscovery }\end{array}$ \\
\hline \multicolumn{4}{|l|}{ Program implementation } \\
\hline Administration time & $2 \mathrm{~h}$ & Simulation center staff & $\begin{array}{l}\text { Scheduling, assigning, and preparing engineers and } \\
\text { other staff for requested layout and rooms needed for } \\
\text { the program }\end{array}$ \\
\hline Participant recruitment & $6-8 \mathrm{~h}$ & Medical or psychosocial clinicians & $\begin{array}{l}\text { Contacting patient families scheduled for spinal fusion } \\
\text { surgery regarding interest in participating in SIM- } \\
\text { Discovery }\end{array}$ \\
\hline Orientation & $30 \min$ & $\begin{array}{l}\text { All key stakeholders involved in } \\
\text { SIMDiscovery (please refer to } \\
\text { Table 1) }\end{array}$ & $\begin{array}{l}\text { Orientation to simulation space, SIMDiscovery } \\
\text { program, and psychological safety prior to first } \\
\text { SIMDiscovery }\end{array}$ \\
\hline Running of SIMDiscovery program & $4 \mathrm{~h}$ & $\begin{array}{l}\text { All key stakeholders involved in } \\
\text { SIMDiscovery (please refer to } \\
\text { Table 1) }\end{array}$ & $\begin{array}{l}\text { Completion of SIMDiscovery program with patients } \\
\text { and families }\end{array}$ \\
\hline
\end{tabular}


Time spent building and operating the program does, indeed, divert time that would otherwise been spent in clinical activities (i.e., seeing patients, conducting surgery); however, key stakeholders were committed to scheduling the program during times of day that were minimally disruptive to clinic and clinical responsibilities. Further, we believe that time dedicated to group preparation could reduce the time spent responding to calls from individual families (i.e., fewer phone calls regarding preparation questions) as well as post-operative questions regarding anxiety or other complications. These are also areas that would benefit from further examination in future studies.

\section{Limitations}

Several limitations exist. The sample size was small as recruitment depended upon families able to attend SIMDiscovery (i.e., 80 families were contacted and 34 attended the program). As such, we offered the intervention to all eligible participants (i.e., 28 families, 22 of which agreed) and did not utilize a control group. Selection bias exists as families chose to participate in SIMDiscovery knowing they would receive enhanced details and information about the surgical procedure - conversely, families may have declined participation in SIMDiscovery due to high levels of anxiety at baseline. Finally, as the sample size was not demographically diverse, it is unclear if we would have yielded similar results with other patient groups.

\section{Future directions}

Despite these limitations, initial results were encouraging. This novel program has the potential to evolve with future research utilizing a randomized control design-comparing SIMDiscovery participants with patients who undergo standard preparation procedures. These studies should examine the impact of SIMDiscovery on additional postoperative outcomes (e.g., improved ambulation, postoperative analgesic use, return to activities). Additional questionnaires for caregivers would be useful in exploring contributors to feelings of anxiety, specifically related to the postoperative period. Future research should also examine patient differences between age cohorts, as well as specific program components that contribute to improved psychological outcomes and those that participants identify as most beneficial. Results from such studies could lead to the expansion of such programs to other medical populations undergoing invasive medical procedures.

To address the attendance barriers observed in this study, alternative ways to participate could be offered. For example, more recently due to the COVID-19 pandemic, SIMDiscovery was offered virtually. Of the 18 families invited to the first virtual session, 14 participated-an attendance rate of $77 \%$. If the resources to run such a program are barriers, institutions can consider offering opportunities for brief, experiential preparation during individual pre-operation appointments as well as adding experiential components to already existing hospital pre-operation programs within Child Life Services departments.

\section{Conclusions}

We created a novel program to help prepare patients and caregivers for surgery. In this preliminary study, patients and caregivers reported decreased anxiety and increased knowledge and preparedness following participation in SIMDiscovery; these changes were generally maintained throughout the post-operative period. Findings support the benefit of programs that expand beyond typical preparation in utilizing hands-on, experiential learning opportunities. Patients and caregivers identified areas in which they desired additional preparation, highlighting the importance of continuing to adapt preparation programs based on families' needs.

Supplementary Information The online version contains supplementary material available at https://doi.org/10.1007/s43390-021-00322-6.

Acknowledgements We thank the following Boston Children's Hospital departments and programs for their ongoing support of the development and facilitation of the clinical SIMDiscovery - Orthopedics program including the Department of Anesthesia, Critical Care and Pain Medicine; the Department of Child Life Services; Perioperative Programs; Surgical Programs; Physical Therapy and Occupational Therapy Services; and the Department of Social Work.

Author contribution All the authors made substantial contributions to the conception or design of the work; or the acquisition, analysis, or interpretation of data; or the creation of new software used in the work; drafted the work or revised it critically for important intellectual content; approved the version to be published; and agree to be accountable for all aspects of the work in ensuring that questions related to the accuracy or integrity of any part of the work are appropriately investigated and resolved.

Funding No funding sources to disclose. No sources of support to disclose.

Data availability De-identified data available on request.

\section{Declarations}

Conflict of interest No conflicts of interest to disclose.

\section{References}

1. Asher MA, Burton DC (2006) Adolescent idiopathic scoliosis: natural history and long term treatment effects. Scoliosis 1(1):2

2. Starkweather AR, Witek-Janusek L, Nockels RP, Peterson J, Mathews HL (2006) Immune function, pain, and 
psychological stress in patients undergoing spinal surgery. Spine 31(18):E641-E647

3. LaMontagne LL, Hepworth JT, Cohen F, Salisbury MH (2003) Cognitive-behavioral intervention effects on adolescents' anxiety and pain following spinal fusion surgery. Nurs Res 52(3):183-190

4. LaMontagne LL, Hepworth JT, Salisbury MH, Riley LP (2003) Optimism, anxiety, and coping in parents of children hospitalized for spinal surgery. Appl Nurs Res 16(4):228-235

5. LaMontagne LL, Hepworth JT, Johnson BD, Cohen F (1996) Children's preoperative coping and its effects on postoperative anxiety and return to normal activity. Nurs Res 45(3):141-147

6. Kain ZN, Caldwell-Andrews AA, Maranets I et al (2004) Preoperative anxiety and emergence delirium and postoperative maladaptive behaviors. Anesth Analg 99(6):1648-1654

7. Kain ZN, Mayes LC, OConnor TZ, Cicchetti DV (1996) Preoperative anxiety in children-predictors and outcomes. Arch Pediat Adol Med 150(12):1238-1245

8. Rhodes L, Nash C, Moisan A et al (2015) Does preoperative orientation and education alleviate anxiety in posterior spinal fusion patients? A prospective, randomized study. J Pediatr Orthop 35(3):276-279

9. Bennett HD, Coleman EA, Parry C, Bodenheimer T, Chen EH (2010) Health coaching for patients with chronic illness. Fam Pract Manag 17(5):24-29

10. Bell RA, Kravitz RL, Thom D, Krupat E, Azari R (2002) Unmet expectations for care and the patient-physician relationship. J Gen Intern Med 17(11):817-824

11. Harris PA, Taylor R, Thielke R, Payne J, Gonzalez N, Conde JG (2009) Research electronic data capture (REDCap)-a metadata-driven methodology and workflow process for providing translational research informatics support. J Biomed Inform 42(2):377-381

12. Spielberger CD (1983) State-trait anxiety inventory for adults

13. Spielberger CD, Edwards CD (1973) State-trait anxiety inventory for children: STAIC: how i feel questionnaire: professional manual: mind garden

14. Julian LJ (2011) Measures of anxiety: state-trait anxiety inventory (STAI), Beck anxiety inventory (BAI), and hospital anxiety and depression scale-anxiety (HADS-A). Arthritis Care Res 63(Suppl 11):S467-S472

15. Bringuier S, Dadure C, Raux O, Dubois A, Picot MC, Capdevila $\mathrm{X}$ (2009) The perioperative validity of the visual analog anxiety scale in children: a discriminant and useful instrument in routine clinical practice to optimize postoperative pain management. Anesth Analg 109(3):737-744

16. Abend R, Dan O, Maoz K, Raz S, Bar-Haim Y (2014) Reliability, validity and sensitivity of a computerized visual analog scale measuring state anxiety. J Behav Ther Exp Psychiatry 45(4):447-453

17. Archbold PG, Stewart BJ, Greenlick MR, Harvath T (1990) Mutuality and preparedness as predictors of caregiver role strain. Res Nurs Health 13(6):375-384

18. Aranha PR, Sams LM, Saldanha P (2017) Impact of multimodal preoperative preparation program on children undergoing surgery. Arch Med Health Sci 5(2):208 